\title{
Pembrolizumab combined with stereotactic body radiotherapy in a patient with human immunodeficiency virus and advanced non-small cell lung cancer: a case report
}

Dongqi $\mathrm{Li}^{1}$, Chuanchun $\mathrm{He}^{1}$, Yaoxiong $\mathrm{Xia}^{2}$, Yaxi Du ${ }^{3}$ and Jing Zhang ${ }^{1 *}$

\begin{abstract}
Background: Pembrolizumab has significantly improved outcomes in patients with advanced non-small cell lung cancer. Combining programmed death-1 inhibitor with stereotactic body radiotherapy showed a slight toxicity and good benefits in recent clinical trials. However, patients infected with human immunodeficiency virus were excluded from most trials because it was assumed that their anti-tumor immunity was compromised compared with immunocompetent patients.
\end{abstract}

Case presentation: In June 2016, a 52-year-old Chinese man presented with human immunodeficiency virus and lung adenocarcinoma (T1 bN3M1b). From November 2016 to December 2016, systemic chemotherapy and palliative radiotherapy for bone metastasis of femoral neck were carried out, but the tumor progressed. In January 2017, after immunochemistry detection of programmed death-1 and programmed death-ligand 1 expression (both > 50\%), pembrolizumab was started. Three weeks after pembrolizumab, we combined stereotactic body radiotherapy for the primary lung tumor. He received no comfort and his CD4 lymphocyte count was stable. Human immunodeficiency virus-ribonucleic acid remained below the limits of detection. In March 2017, after three cycles of pembrolizumab and 5 weeks of stereotactic body radiotherapy therapy, he suddenly presented with palpitations. Emergency computed tomography scanning showed massive pericardial effusion and interstitial pneumonia. So we interrupted the pembrolizumab use and initiated treatment with prednisolone $1 \mathrm{mg} / \mathrm{kg}$; however, the tumor progressed. Then, his CD4 lymphocyte count declined. Finally he died in June 2017 due to dyscrasia.

Conclusions: Pembrolizumab combined with SBRT therapy for patients with human immunodeficiency virus infection and non-small cell lung cancer may lead to serious immune-related adverse events and more clinical trials are needed.

Keywords: Pembrolizumab, Stereotactic body radiotherapy, Immune-related adverse events, Non-small cell lung cancer, HIV

\section{Background}

Pembrolizumab, the first Food and Drug Administration (FDA)-approved programmed death-1 (PD-1) inhibitor, has significantly improved outcomes in patients with advanced non-small cell lung cancer (NSCLC) [1].Combining PD-1 inhibitor with stereotactic body radiotherapy
(SBRT) showed a slight toxicity and good benefits in recent clinical trials $[2,3]$. However, patients with human immunodeficiency virus (HIV) infection were excluded from most trials, because it was assumed that their anti-tumor immunity was compromised compared with immunocompetent patients.

\section{Case presentation}

${ }^{1}$ Bone and Soft Tissue Tumors Research Center of Yunnan Province, Department of Orthopaedics, The Third Affiliated Hospital of Kunming Medical University (Tumor Hospital of Yunnan Province), Kunming, Yunnan 650118, People's Republic of China

Full list of author information is available at the end of the article
Here, we report the case of a patient with HIV and advanced NSCLC who was treated with PD-1 inhibitor (pembrolizumab) combined with SBRT. 
In June 2016, a 52-year-old Chinese man who had never smoked tobacco, who had an initial diagnosis of HIV infection in 2013 with highly active antiretroviral therapy, was diagnosed as having advanced lung adenocarcinoma (T1bN3M1b) with KRAS mutation (exon 2 deletion). From November 2016 to December 2016, systemic chemotherapy (carboplatin/ pemetrexed for two cycles) and palliative radiotherapy for bone metastasis of femoral neck (intensity-modulated radiation therapy, 48 Gy/16 fractions) were carried out, but the tumor progressed with new metastatic lymph nodes (Fig. 1a).
In January 2017, after immunochemistry detection of PD-1 and programmed death-ligand 1 (PD-L1) expression (both $>50 \%$, Fig. 1b), pembrolizumab was started ( $2 \mathrm{mg} / \mathrm{kg}$, every 3 weeks). Three weeks after pembrolizumab, we combined SBRT for the primary lung tumor (50 Gy/five fractions, every other day). Our patient received no comfort and his CD4 lymphocyte count was stable. Human immunodeficiency virus-ribonucleic acid (HIV-RNA) remained below the limits of detection.

In March 2017, after three cycles of the pembrolizumab and 5 weeks of SBRT therapy, he suddenly presented with palpitations. Emergency computed tomography (CT)

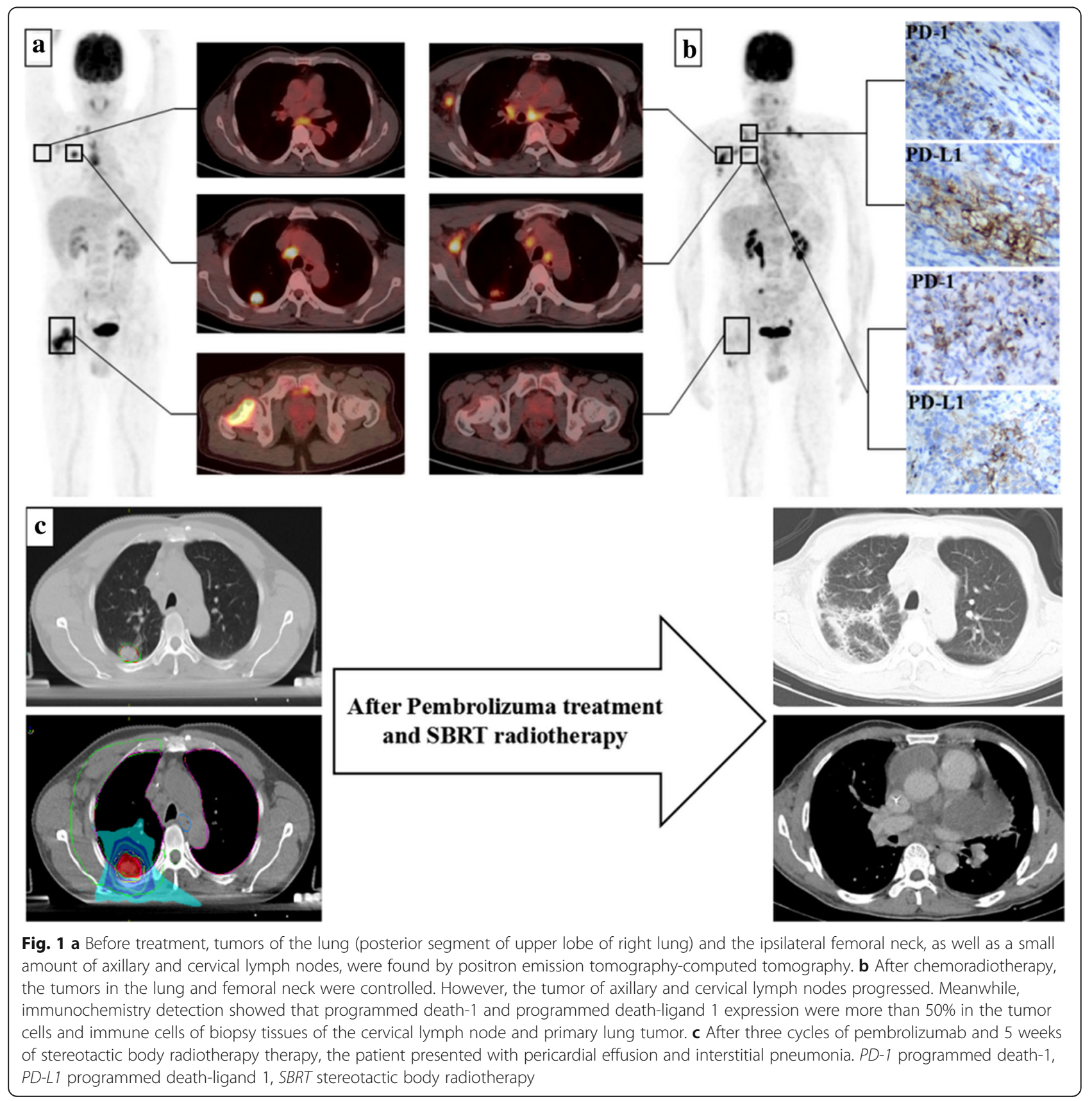


scanning showed massive pericardial effusion and interstitial pneumonia (Fig. 1c). So we interrupted the pembrolizumab use and initiated treatment with prednisolone $1 \mathrm{mg} / \mathrm{kg}$; however, the tumor progressed. Then, his CD4 lymphocyte count declined. Finally he died in June 2017 due to dyscrasia.

\section{Discussion}

The use of highly active anti-retroviral therapy has prolonged the survival of patients with HIV, which increased the incidence of HIV-related malignancies, including lung cancer [4]. The treatment of patients with HIV infection and NSCLC has rapidly gained attention. Both mechanisms of pembrolizumab and SBRT therapy aroused anti-tumor effect of immunity cells [5]. To our knowledge, this is the first case report on treating patients with HIV infection and NSCLC by combining pembrolizumab with SBRT consecutively.

The latest two case reports on PD-1 inhibitor in treating patients with HIV infection and NSCLC were inconsistent. Regarding the tumor response, one case had complete remission [6], but the other progressed [7]. Our results were in line with the latter. One reason might be that the anti-tumor effect of immunity cells was weakened after chemoradiotherapy. We also thought that impaired bone marrow reserve resulting from chemoradiotherapy may have contributed to the CD4 cell decline.

It seems that HIV status has no impact on the local tumor immune microenvironment, including immune cell subset (CD3, CD4, CD8, and CD68) infiltration or PD-L1 expression [8]. However, it is difficult to explain the mechanism of rapid immune-related adverse events (IRAEs). The latest retrospective controlled study $(n=164)$ showed that thoracic radiotherapy did not increase the risk of interstitial pneumonia [9]. In this case, we report other IRAEs of pericardial effusion. It is essential to select defining biomarkers that predict immunotherapy response and IRAEs [10].

\section{Conclusion}

Pembrolizumab combined with SBRT therapy for patients with HIV infection and NSCLC may lead to serious IRAEs and more clinical trials are needed.

\footnotetext{
Abbreviations

IRAEs: Immune-related adverse events; NSCLC: Non-small cell lung cancer; PD-1: Programmed death-1; PD-L1: Programmed death-ligand 1; SBRT: Stereotactic body radiotherapy

\section{Acknowledgements}

Not applicable.

\section{Funding}

This work was supported by the National Natural Science Foundation of China (grant numbers 81302343) and the National Natural Science Foundation of China (grant numbers 81760486).
}

Availability of data and materials Not applicable.
Authors' contributions

All authors contributed in the diagnosis and treatment of the patient and in preparing the final manuscript. All authors read and approved the final manuscript.

Ethics approval and consent to participate Not applicable.

\section{Consent for publication}

Written informed consent was obtained from the patient's wife for publication of this case report and any accompanying images. A copy of the written consent is available for review by the Editor-in-Chief of this journal.

\section{Competing interests}

The authors declare that they have no competing interests.

\section{Publisher's Note}

Springer Nature remains neutral with regard to jurisdictional claims in published maps and institutional affiliations.

\section{Author details}

'Bone and Soft Tissue Tumors Research Center of Yunnan Province, Department of Orthopaedics, The Third Affiliated Hospital of Kunming Medical University (Tumor Hospital of Yunnan Province), Kunming, Yunnan 650118, People's Republic of China. ${ }^{2}$ Department of Radiotherapy, The Third Affiliated Hospital of Kunming Medical University (Tumor Hospital of Yunnan Province), Kunming, Yunnan 650118, People's Republic of China. ${ }^{3}$ Lung Cancer Research Center, The Third Affiliated Hospital of Kunming Medical University (Tumor Hospital of Yunnan Province), Kunming, Yunnan 650118, People's Republic of China.

Received: 10 January 2018 Accepted: 23 March 2018

Published online: 23 April 2018

\section{References}

1. Reck M, Rodríguez-Abreu D, Robinson AG, Hui R, Csőszi T, Fülöp A, Gottfried M, Peled N, Tafreshi A, Cuffe S, O'Brien M, Rao S, Hotta K, Leiby MA, Lubiniecki GM, Shentu Y, Rangwala R, Brahmer JR. Pembrolizumab versus Chemotherapy for PD-L1-Positive Non-Small-Cell Lung Cancer. N Engl J Med. 2016;375:1823-33.

2. Sundahl N, De Wolf K, Rottey S, Decaestecker K, De Maeseneer D, Meireson A, Goetghebeur E, Fonteyne V, Verbeke S, De Visschere P, Reynders D, Van Gele M, Brochez L, Ost P. A phase I/II trial of fixed-dose stereotactic body radiotherapy with sequential or concurrent pembrolizumab in metastatic urothelial carcinoma: evaluation of safety and clinical and immunologic response. J Transl Med. 2017;15:150.

3. De Wolf K, Kruse V, Sundahl N, van Gele M, Chevolet I, Speeckaert R, Brochez L, Ost P. A phase II trial of stereotactic body radiotherapy with concurrent anti-PD1 treatment in metastatic melanoma: evaluation of clinical and immunologic response. J Transl Med. 2017;15(1):21.

4. Sigel K, Makinson A, Thaler J. Lung cancer in persons with HIV. Curr Opin HIV AIDS. 2017;12:31-8.

5. Herrera FG, Bourhis J, Coukos G. Radiotherapy combination opportunities leveraging immunity for the next oncology practice. CA Cancer J Clin. 2017;67:65-85.

6. Mccullar B, Alloway T, Martin M. Durable complete response to nivolumab in a patient with HIV and metastatic non-small cell lung cancer. J Thorac Dis. 2017;9:E540-2.

7. Hentrich $M$, Schipek-Voigt $K$, Jäger $H$, et al. Nivolumab in HIV-related non-small-cell lung cancer. Ann Oncol. 2017:28:2890.

8. Yanik EL, Kaunitz GJ, Cottrell TR, Succaria F, McMiller TL, Ascierto ML, Esandrio J, Xu H, Ogurtsova A, Cornish T, Lipson EJ, Topalian SL, Engels EA, Taube JM. Association of HIV Status with Local Immune Response to Anal Squamous Cell Carcinoma: Implications for Immunotherapy. JAMA Oncol. 2017;3:974-8.

9. Hwang WL, Niemierko A, Willers H, Keane FK, Justin F. Immune-related adverse events (IRAEs) in metastatic lung cancer patients receiving PD-1/ PD-L1 inhibitors and thoracic radiotherapy. J Clin Oncol. 2017;35:9079.

10. Nishino M, Ramaiya NH, Hatabu H, Hodi FS. Monitoring immune-checkpoint blockade: response evaluation and biomarker development. Nat Rev Clin Oncol. 2017;14(11):655-68. 